\section{Mars still Mysterious}

The quality of the first Mariner 6 close-ups of Mars to come off the monitors at the Jet Propulsion Laboratory seem to match the first releases of Orbiter pictures of the Moon, and there is hope that, with electronic processing to remove transmission noise and enhance surface features, the pictures will be much improved. Earlier this week, the indications were that the two probes were up to scratch, with Mariner 6 having produced most if not all of the expected series of seventy pictures. So far, at least, there seem to have been few surprises. Planetary scientists were prepared for extensive cratering by the Mariner 4 pictures four years ago, and the appearance of the south polar cap is much as expected. The ragged edge of the cap (Fig. 2) has already been noted from telescope observations and is thought to be due to height differences. The abundance of carbon dioxide in the atmosphere indicates that the pole caps are probably carbon dioxide rather than ice, but infrared scanning has picked up indications of water, thought by JPL to be ice crystals in an ice fog.

Fig. 1 is one of the close-up pictures, taken when Mariner 6 was at an altitude of 2,150 miles, and covers an area roughly 560 miles by 430 miles. So far there is no indication where on Mars these heavily eroded craters are. With the presence of an atmosphere and possible erosion of surface features by dust storms, debate on the age and origin of the surface of Mars is likely to be more involved than that of the Moon. Now that there seems to be evidence for a molten interior to the seemingly dead Moon, tectonic forces will probably also have to be included in the discussions of Mars, and the proximity to the asteroid belt means that the rate of meteorite impact could be quite different for the two bodies. But many of the small meteorites which rain down on the Moon could be burnt up in the martian atmosphere.

The craters in Fig. 3 are sharp, indicating comparatively recent bombardment. The picture is an enlargement from one of the Mariner 6 close-ups and the largest crater is about 4.5 miles across. Fig. 4 covers an area of 52 miles by 45 miles, showing a large crater 15 miles in diameter.

Although the Mariner pictures are expected to have a best resolution of about 900 feet when processing is complete, there is as yet no indication of linear features which could be the legendary canals. Current thought is that the canals are an artefact of the eye, which tends to join together separate small features which lie on a line, and that the small features are tectonically controlled.

Continued overleaf

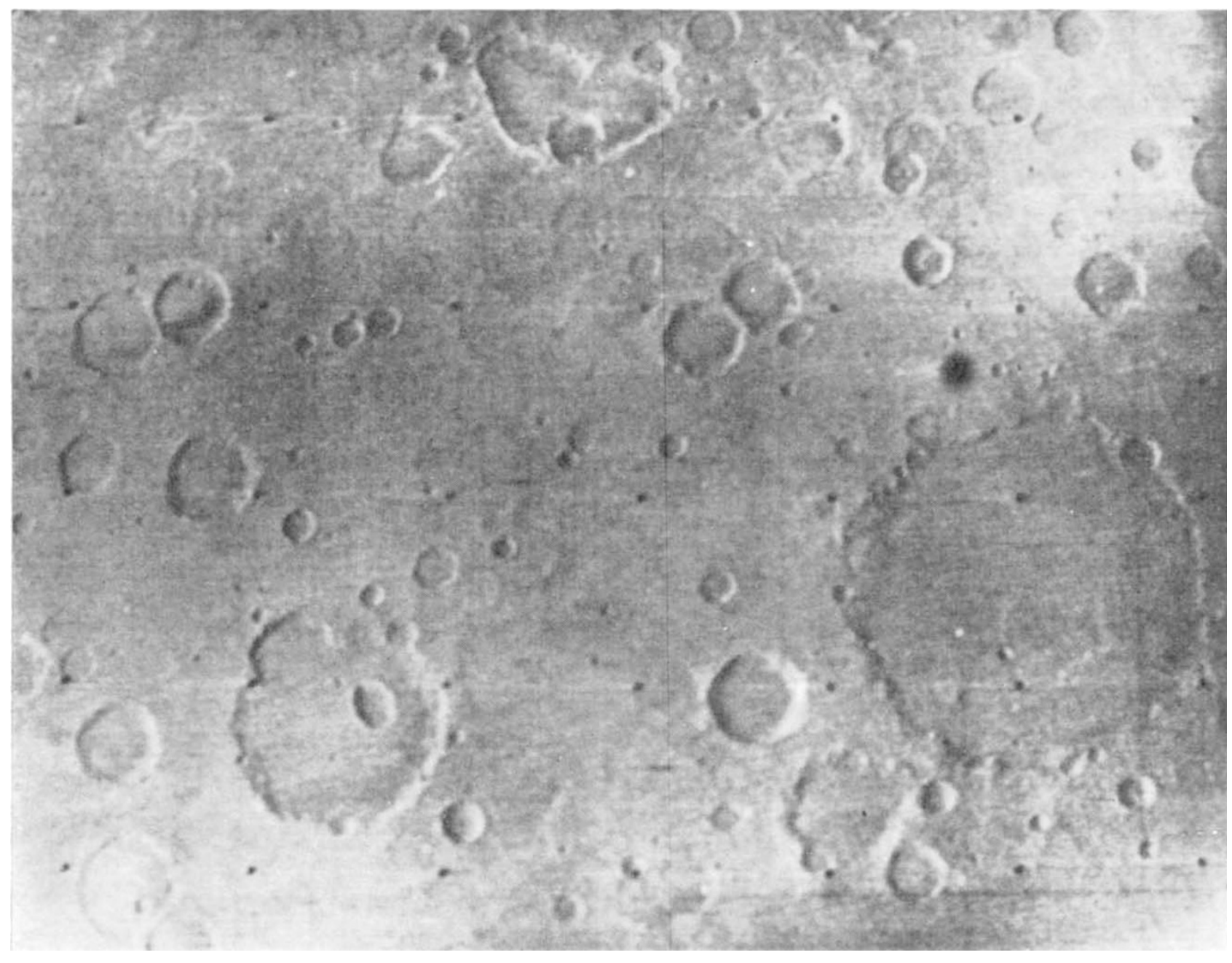

Fig. I. Mars from 2,150 miles. 
The chief surprise so far, and ominous for any hope of finding life, is the absence of nitrogen in the martian atmosphere. Nitrogen might have been expected as a result of volcanic activity. But the infrared spectrometer is said to have hinted at the presence of hydrocarbons.

\section{New Radar Map}

Hours before Mariner 6 began taking approach pictures of Mars last week, new radar measurements of the planet's equatorial zone were reported by the Jet Propulsion Laboratory of the California Institute of Technology which is running the current Mariner project. According to the new figures, regions along the martian equator differ in height by as much as 8.3 miles. The measurements were made with the Jet Propulsion Laboratory's 210 foot steerable dish aerial at Goldstone in California, which took part in the Apollo 11 mission and has been tracking the two Mars probes, Mariner 6 and 7 . In a series of radar observations from May 7 until the day before the launch of Apollo 11, bracketing the time of closest approach of Mars on June 9, JPL investigated fifteen locations in a band extending from the equator to latitude $10^{\circ}$ north. Because of the width of the radar beam, each

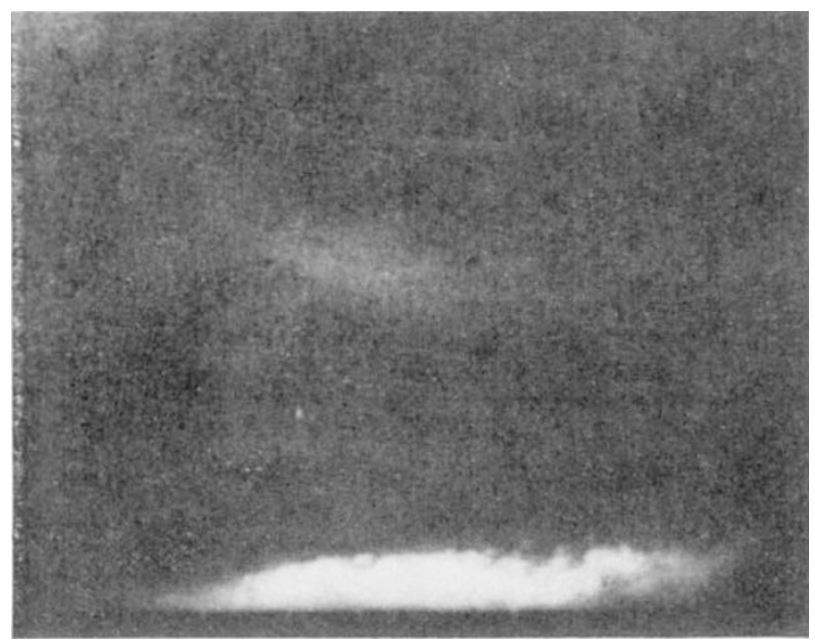

Fig. 2. The forty-seventh picture in the Mariner 6 approach sequence, showing the polar cap south of Syrtis Major. The ragged edge of the cap and a semicircular feature perhaps representing a crater are visible.

spot covered 3,700 square miles, so that individual peaks and craters could not be distinguished. Instead the JPL work shows the gencral trend of altitude in the equatorial band-the region photographed in close-up by Mariner 6, for example, seems to be relatively flat.

The height difference of 8.3 miles seems to be between the martian equatorial regions Tharsis and Amazonis. Other results are that two dark areas, Syrtis Major and Trivium Charontis, are sloping regions, Syrtis Major rising gently over 500 miles to the plateau. Aeria at about 20,000 feet. Between Xanthe and Meridiani Sinus, the equatorial band covered by Mariner, there is a gentle slope downward toward the east, with a total height change of about a mile.

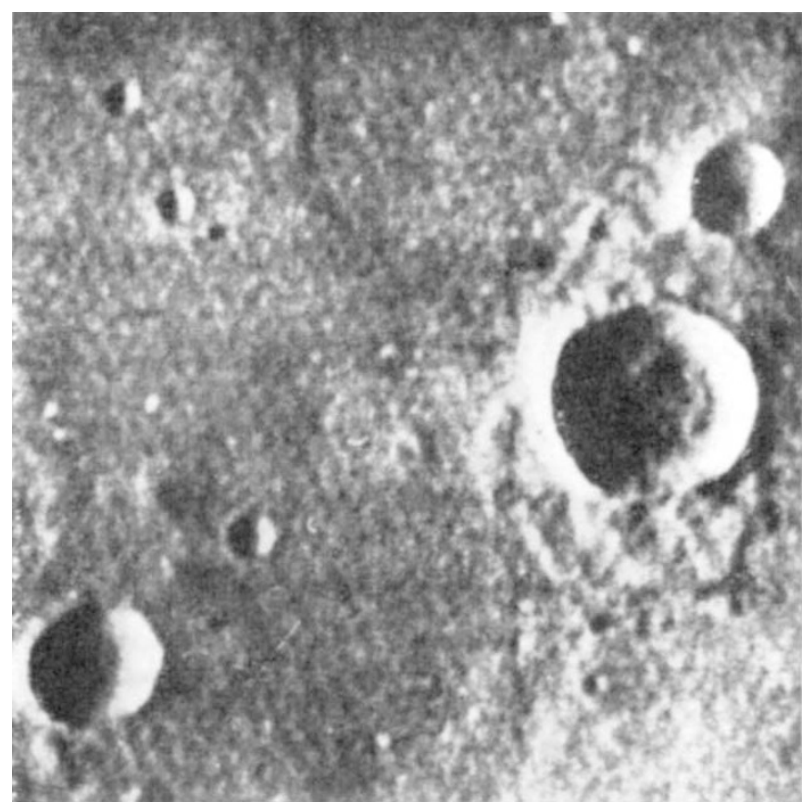

Fig. 3. Part of one of the close-up sequences. The two larger craters are 4.5 miles and 3 miles in diameter.

The chief conclusion from the radar mapping is that the colour of the martian surface is not a perfect indicator of height. Previously scientists have been divided about whether the light areas represent highlands or lowlands, but the radar results show that light areas can be either highlands or lowlands, while the dark areas seem to be somewhere in between. The measurements were carried out at $12.5 \mathrm{~cm}$, using a transmitter power of 450 kilowatts. The JPL points out that if ocean trenches on the Earth are taken into account, the height differences of 8.3 miles on Mars match the differences of 12 miles encountered on the Earth.

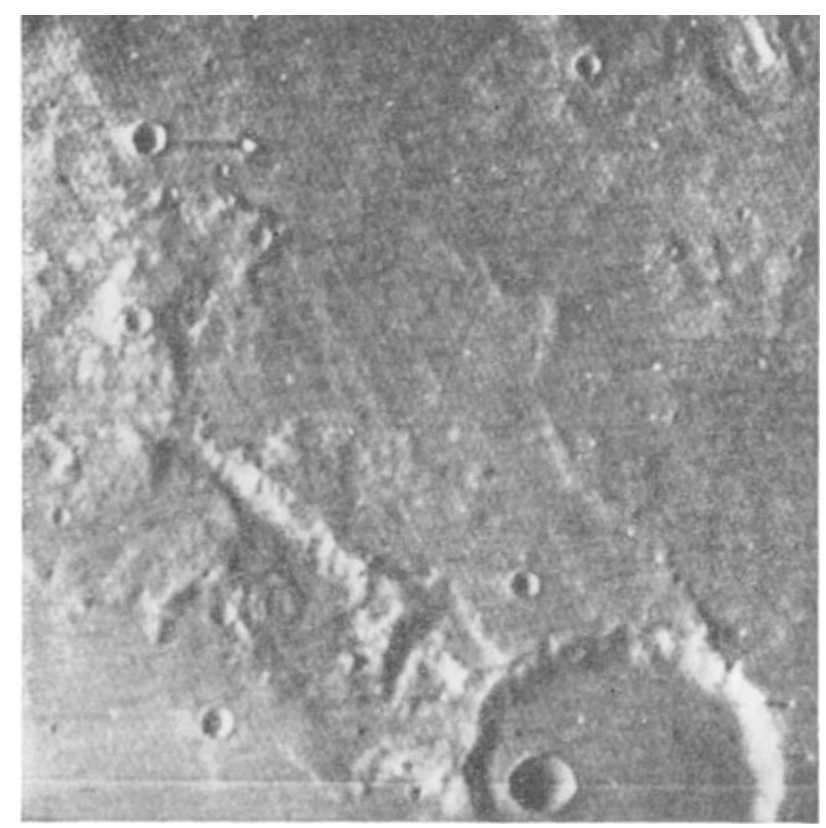

Fig. 4. A close-up view covering an area of 52 miles by 45 miles. 